\title{
Contextual Analysis - a Multiperspective Inquiry into Emergence of Complex Socio-cultural Systems
}

Peter M. Bednar,

School of Computing, University of Portsmouth, UK;

Department of Informatics, Lund University, Sweden.

Email: peter.bednar@ics.lu.se

\begin{abstract}
This paper explores the concept of organizations as complex human activity systems, through the perspectives of alternative systemic models. The impact of alternative models on perception of individual and organizational emergence is highlighted. Using information systems development as an example of management activity, individual and collective sense-making and learning processes are discussed. Their roles in relation to information systems concepts are examined. The main focus of the paper is on individual emergence in the context of organizational systems. A case is made for the importance of attending to individual uniqueness and contextual dependency when carrying out organizational analyses, e.g. information systems analysis. One particular method for contextual inquiry, the framework for Strategic Systemic Thinking, is then introduced. The framework supports stakeholders to own and control their own analyses. This approach provides a vehicle through which multiple levels of contextual dependencies can be explored and allows for individual emergence to develop.
\end{abstract}

Keywords: Strategic Systemic Thinking, Contextual Analysis, Individual Emergence, Contextual Dependency. 


\section{Introduction}

The call for papers for the AIRS Congress 2007 (Minati, 2006) suggests that a study of processes of emergence implies a need to model and distinguish the establishment of structures, systems and systemic properties. It goes on to point out that, in a constructivist view, an observer identifies such properties by application of models. Different perceptions of structures and systems correspond to different, irreducible models. Perceived emergence of systemic properties, e.g. functionality in computer systems or collective learning abilities in social systems, then ensues from application of such models. The author of this paper wishes to compare and contrast two alternative models that may be applied in forming constructivist views of organizational systems. The paper shows how one particular model highlights the importance of individual, as well as organizational emergence. Its contribution is to argue for a move away from reductionist cybernetic models towards critical systemic thinking - from attempts to reduce uncertainties inherent in management of organizations towards approaches which embrace 'complexification.' Using information systems development as an example, the implications for individual and collective learning in organizations are explored and a case for contextual methods of inquiry to support organizational learning is made. A particular framework for contextual inquiry is then described in outline.

An organisation may be viewed as a complex social system, affected by goals and values of the individuals within it (Schein 1992). We are reminded by Senge (1990) that "Today, systems thinking is needed more than ever because we are becoming overwhelmed by complexity. Perhaps for the first time in history, humankind has the capacity to create far more information than anyone can absorb, to foster far greater interdependency than anyone can manage, and to accelerate change far faster than anyone's ability to keep pace....organizations break down, despite individual brilliance and innovative products, because they are unable to pull their diverse functions and talents into a productive whole." (Senge, 1990, p69)

The nature of these social systems, their sub-systemic structures and the relations which sustain them over time vary widely from one organization to another. An organization can also be viewed as a purposeful human activity system (Checkland 1999). However, objective agreement on the nature of such systems is elusive, since the defining properties of 'the system' will depend upon the viewpoint of the individual who considers it. For example, when a person enters a bank as a customer, he is likely to view this organization as a system for providing him with financial services. However, to a person who enters that bank as an employee, it may appear to be a system for providing her with a livelihood. (Checkland refers to these differing perspectives as 'Weltanschauungen' or 'worldviews'). Schein (1992) suggested that organizational culture is formed over time through shared goals. Such sharing could only be achieved through a negotiation of differing perspectives held by individuals what Checkland refers to as Weltanshauungen. For this reason, agreement on a single description of a 'real' human activity system will remain elusive and consensus on its goals difficult to achieve.

Within any 'organization', an interacting collection of living individuals can be found, each with a unique life history and worldview. Every individual produce her/his own unique understanding of context, constructed through interaction with organizational 
systems and environment by means of a variety of sense-making strategies (Weick, 1995; Bateson, 1972; Berger and Luckman, 1966). Those taking on responsibility for management as an activity need to be aware of the challenges posed by these differing perspectives. One possible definition of 'management' is 'a set of practices and discourses embedded within broader asymmetrical power relations, which systematically privilege the interests and viewpoints of some groups, whilst silencing and marginalizing others.' (Levy et al (1998), from Alvesson and Willmott (1996)).

Langefors (1995) discusses the role of organizational information systems. He considered that, in order to manage an organization, it would be necessary to know something about the current state and behaviour of its different parts and also the environment within which it was interacting. These parts would need to be coordinated and inter-related, i.e. to form a system. Thus, means to obtain information from the different parts of a business would be essential and these means (information units) would also need to be inter-related. Since the effectiveness of the organization would depend upon the effectiveness of the information units, an organization could be seen as crucially 'tied-together' by information. For Langefors, therefore, the organization and its information system could be viewed as one and the same.

The next section of the paper sets out some of the theoretical background within which contemporary systemic models have been framed. This is followed by a discussion of learning and knowing in an organizational context. Contrasting models of organizational systems are then set out, showing how different perspectives on emergence result from their application. A role for contextual inquiry in enabling individual, as well as organizational emergence to be explored is then set out. One possible method of contextual inquiry is explained. The final section of the paper attempts to summarise the arguments.

\section{Background}

Many attempts have been made in the past to understand and manipulate social phenomena by application of laws derived from the natural world. Ackoff (1999) quotes examples set out by sociologist Sorokin (1928) where researchers had attempted to establish laws of 'social physics'. He also notes that philosopher Herbert Spencer referred to a general characteristics of 'life' (accepted in relation to biological phenomena) as no less applicable to society, i.e. characteristics of growth, increasing differentiation of structure and increasing definition of function. A great deal of research is available on systems perspectives in social science (see for example West Churchman, 1968; Simon, 1979). However, as Emery (1969) points out, these contributions have been fragmented and diverse, often using similar terms to denote quite different concepts. Attempts have been made to liken the operation of social 'systems' to mechanistic models derived from engineering (see, for example, applications of the Shannon-Weaver model from telecommunications to human interaction and communication) or to organic models from biology (e.g. applications of Maturana and Varela's theory of autopoeisis). Ulrich (1983) provides a discussion of the way that root metaphors in systems thinking influence the way in which a person conceives of 'a system'. Without these metaphors, the concept of a system might have remained 'empty.'

The scope for systemic research to inform management thinking has therefore been diverse and confused. Perhaps one of the most influential works has been the General 
Systems Theory of Von Bertalanffy (1968). He did not favour direct application of mechanistic models to human problems, suggesting instead: '... systems science, centered in computer technology, cybernetics, automation and systems engineering, appears to make the systems idea into another - and indeed the ultimate - technique to shape man and society ever more into the "mega machine ..."'(1968, p viii). In his chapter on 'The Meaning of General Systems Theory' he points out that models which are essentially quantitative in nature have limited application to phenomena where qualitative interpretations 'may lead to interesting consequences' (p.47). Nevertheless, cybernetic models derived from GST have had great appeal in management literature. In particular, a concept of sub-optimality has been the focus of attention. Boulding (1953), for instance, attempts to establish laws of organization. His law of instability suggests that organizations fail to reach a stable equilibrium in relation to their goals due to cyclic fluctuations resulting from the interaction of subsystems. Ways to remove sub-optimality, a result of conflict between systemic and sub-systemic goals, have therefore been identified as a key function of management as it attempts Fayol's classic tasks of planning, directing and controlling (Fayol, 1949). The reflection is that learning must surely be a prerequisite to purposeful activities of the kind Fayol describes. Bateson (2003) reminds us that a critical element of learning is reflexivity - awareness of one's own responses to context. Such reflexivity should inform any systemic view of human activities.

From an interpretive perspective, an individual's sense-making is co-dependent with the organizational culture within which it takes place, and requires continual construction/re-construction through reflection over time (Schein 1992). A perception of organizational life focused on goal-seeking is therefore problematic. Vickers (1970) argues that life consists in experiencing relations rather than seeking 'ends'. He challenges a cybernetic paradigm which a goal-seeking model implies, suggesting instead a cyclical process in which experience generates individual norms and values. These in turn create a readiness in people to notice aspects of their situation, measure them against norms and discriminate between them. Our 'appreciative settings' condition our perceptions of new experiences, but are also modified by them. Development of an individual's appreciative system is thus ongoing over time as a backdrop to social life. If individual sense-making is co-dependent with organizational culture there must be some interaction between them, built on communication.

Information can be defined as data which is rendered meaningful in a particular context. The meaning attributed to an item may well vary when understood from the point of view of different individuals. Each individual produces her/his own understanding of contexts within which information is formed, constructed through interaction with organizational systems and their environment by means of a variety of sense-making strategies (Weick 1995). During the 1960's, Borje Langefors developed the 'Infological Equation' (see Langefors, 1966). This work identifies the significance of interpretations made by unique individuals within specific organizational contexts (e.g. Langefors, 1995). The Infological Equation " $I=i(D, S, t)$ " shows how meaningful information (I) may be constructed from the data (D) in the light of participants' pre-knowledge (S) by an interpretive process (i) during the time interval $(\mathrm{t})$. The necessary pre-knowledge (s) is generated through the entire previous life experience of the individual. Individuals perform different systemic roles within organizations, and have unique perspectives derived from the sum of previous life 
experiences. Meanings are constructed by different individuals reflecting their unique world views. While it is possible to construct a 'conduit' through which data may flow around an organization, information is constructed by individuals in their interactions within the organizational context. Logically, therefore, it is possible to develop a data system to support management tasks, but this could only become an information system through direct and interpretive participation from those individuals using it. The logic demonstrated by the Infological Equation suggests that individual learning and organizational development are inextricably bound together. Information systems must therefore provide support for contextually relevant individual learning, and organizational analysis drawing on this learning, as a systemic process over time (Bednar, 2000).

\section{Learning and Knowing}

Those theories that an individual creates through sense-making will be influenced by multiple contextual dependencies arising from her/his experience and environment (Bednar, 2000). Such dependencies have been derived through the particular experiences of individuals involved, in the context of their own working situations. The distinctiveness of each work situation lies in construction of meanings that individuals attach to it. In relation to systems design in particular, therefore, there is no reason to assume consensus among the different actors as to the desirable properties of a proposed system. Indeed, as the Infological Equation demonstrates (see Langefors, 1966), it is not possible for any individual to know in advance precisely what requirements she/he might have. Instead, actors need support to engage in a collaborative endeavour of requirement shaping. Here individuals partake in a learning spiral through reflection on sense-making in a work context in order to create understanding of those emergent 'systems' in their minds.

Individual learning may be described as taking place through sense-making processes as a response to messy and uncertain contexts in which resolutions are sought. Different orders of learning may be identified, based on a cycle of experience and reflection on experience (Argyris and Schon, 1978; Bateson, 1972). Higher orders of learning involve reflection on sense-making processes themselves, i.e. a learning cycle transforms into a spiral. Reflection on sense-making becomes an exercise in practical philosophy. Certain points follow from this. If individual learning is a creative process based in sense-making, then context is clearly important. Any unique individual's view is based in reflection on experience (Bateson, 1972), and experience is context specific. Therefore, an examination of contextual dependencies, as part of analysis, will be important.

Knowing, as a creative process, is inextricably linked to learning. Bateson (1972) suggests that information may be defined as 'a difference that makes a difference', existing only in relation to a mental process. This process is what leads to an individual 'knowing'. Bateson describes a hierarchy of different orders of learning. At level zero, learning represents no change, since the same criteria will be used and reused without reflection. This is the case in rote learning of dates, code words, etc which is contextually independent and in which repeated instances of the same stimuli produce the same resulting 'product'. All other learning, according to Bateson's hierarchy, involves some element of trial and error and reflection. Orders of learning can be classified according to types of errors and the processes by which correction is 
achieved. Level I involves some revision using a set of alternatives within a repeatable context, level II represents revision based on revision of context, and so on. Bateson's hierarchy finds an echo in the work of Argyris and Schon (single and double-loop learning). Double loop learning comes about through reflection on learning processes in which individuals may attempt to challenge prejudices and assumptions arising from their experiences. (Argyris, 1990; Argyris and Schon, 1996). When individuals need to solve an immediate problem, i.e. close a perceived gap between expected and actual experience, they may harness their sense-making processes within contexts of existing goals, values, plans and rules (Vickers's appreciative settings), without questioning their appropriateness. However, if individuals challenge received wisdom and critically appraise assumptions previously applied, double-loop learning occurs. The resulting process creates a productive learning spiral, which is at the heart of any successful organizational innovation.

As mentioned previously, the Infological Equation (Langefors, 1966) suggests that individuals develop unique understandings (meaningful information) by examining data in the light of (their own) pre-knowledge gained from reflecting on experience during a previous time interval. Information, and 'knowledge' derived from it, cannot therefore be seen as commodities, to be transmitted from one individual to another (or stored) as containers of objective meaning. Furthermore, it is through these processes of constructing new understandings/meaning, by examining data in light of experience, that organizations, their goals and cultures are constituted. If individual learning is a creative process, organizational learning is so also.

\section{Complexification and Emergence}

Attempts by students of management to reduce organizational problems to consideration of 'sub-optimality', drawing on mechanistic models from systems science can be seen as reductionism. Exploration of multiple levels of contextual dependency may help analysts to avoid entrapment in various types of reductionism, including undue reliance on sociological, psychological or technological concepts. It may also help to eliminate tendencies towards generalization, or substitution of an external analyst's own views for those of the participating stakeholders. A need to promote deep understandings of problem spaces requires us to go beyond grounding of research in phenomenological paradigms. In order to avoid various types of reductionism and achieve deepened understanding, analysts must attempt to incorporate philosophy as an integral part of their research practice (e.g. Bateson, 1972; Klein, 2007; Nissen, 2007; Ulrich, 1983).

As pointed out by Werner Ulrich in his discussion of boundary critique perception of a system varies with the stance of the observer (Ulrich, 2001), i.e. this differentiates between an observer's and an actor's picture of reality, which means that anyone wishing to inquire into IS use must continually align themselves with actor perspectives. For example, meaning shaping in particular situations can be described through comparisons of different actors' perspectives within given structural criteria, or 'circling of realities'. This refers to a necessity to acquire a number of different perspectives (in time-space) in order to be able to get a better and more stable appreciation of an actor reality (Bednar and Welch, 2007). The whole person includes dimensions of both 'heart' and 'mind' (Ciborra, 2004). Personal perspectives which transcend received, organizational 'common sense thinking' may be encouraged to 
emerge through methods which emphasise individual uniqueness and contextual dependency.

Those engaged in management tasks such as IS design should not forget that they set up personal boundaries for a situation by defining it from their own experiences and preferences. As human beings we all have pre-understandings of phenomena, which are influenced by our own values, 'wishful thinking', and how each of us has been socialized into a particular society. These pre-understandings are being reviewed gradually, with the support of our experience. In a continual exchange/interchange between an individual's pre-understanding and experience, a process of inquiry may progress. It follows from the preceding discussion that, from the point of view of each individual's perception, an organization is an emergent property of inter-individual sense-making processes and activities. The organization is continually constructed/reconstructed for each individual as a result of emergence from individual sense-making perspectives. A critically informed approach to research involves recognition / understanding of this emergence. Without recognition of the uniqueness of each particular individual's experience of organizational life this critical approach may be undermined. Within a traditional scientific paradigm, the focus of a researcher's attention rests on increasing the precision and clarity with which a problem situation may be expressed. This can lead to an artificial separation of theory from praxis, of observation from observer and observed. 'Knowing' about organizational context (formed by on-going construction of meanings through synthesis of new data with past experience) may be deeply embedded and inaccessible to individuals concerned. The perspective promoted in this paper emphasises self-awareness of human individuals. In research undertaken from this perspective, a focus towards emancipation and transparency, rather than clarity and precision, is adopted. A researcher taking such a perspective will recognize that there are uncertainties and ambiguities inherent in socially constructed everyday world views (a similar discussion can be found in Radnitzky, 1970).

In some approaches, a human activity system is regarded as a mental construct derived from an interrelated set of elements, in which the whole has properties greater than the combination of component elements. When such a model is adopted, individual uniqueness is subsumed in perceived emergent properties of a conceptualised system. Even when considered as a duality seen as a system to be served and a serving system (e.g. Checkland and Holwell, 1998), individuals remain invisible. In order to take into account unique individual sense-making processes within an organizational problem arena, there is a need for analysts to explore multiple levels of contextual dependencies. Every observation is made from the point of view of a particular observer (Radnitzky, 1970). Since it is not possible to explore problem spaces from someone else's point of view, it follows that external analysts can only play supportive roles in enabling individuals within given contexts to explore their own sense-making. In an alternative model (de Zeeuw, 2007; Bednar, 2007), an organizational system may be seen as an emergent property of unique, individual sense-making processes and interactions within a particular problem arena. When considered in this way, it is possible to perceive some individuals themselves to have emergent properties of their own which can be larger than (e.g. outside of) those of one particular organizational system seen as a whole. Consider, for instance, a football club seeking to recruit skilful players for its team. The manager may perceive a need for a creative, attacking midfielder to play a role as one component part of the 
team's efforts to win. The Los Angeles Galaxy Club recently experienced such a need but chose to recruit former England captain, David Beckham. Beckham can play the role of an attacking mid-fielder for the team. However, he brings with him qualities which transcend this in terms of his personal notoriety, publicity potential and marketing value for sales of Club products such as replica shirts, etc. Beckham has emergent properties beyond those of any other mid-field footballer in relation to the human activity system which is that Club. This model is not, of course, the same as a non-systemic, fragmented view which focuses on individuals but fails to perceive an emergent system arising through their interactions, and hence ignores the impact of norms, values, expectations, communicational acts, etc. on individual sense-making processes (Hay, 2007).

\section{Contextual Inquiry}

The importance of context for systemic analysis has been widely recognized (see, e.g. Checkland, 1981 or Ulrich, 1983). Contextual inquiry, as described here, is viewed as a special case of contextual analysis. This paper describes an application of a framework for contextual inquiry, the Strategic Systemic Thinking (SST) framework (Bednar, 2000). This forms an exploration into the nature of open systems thinking and how systemic identities are maintained and generated within a specific human activity context. SST maintains a particular focus on ways in which human analysts can deal with complexification and uncertainty although this poses apparently insuperable epistemological problems. Particular emphasis is placed on a multiplicity of individual sense-making processes and ways these are played out within organizations. SST can support groups of organizational actors to take contextual dependencies into consideration, and is intended as a means to enable them to cope with escalations in complexity. A cardinal principle of the framework is that actors should own and control their own inquiry, supported but not dominated by a facilitating professional analyst.

When an attempt is made to evaluate effectiveness in managing or 'designing' organizational systems, concepts of analysis become important. Good practice requires an understanding that addresses intrinsic and contextually-dependent characteristics of organizational activities. An understanding can only come about through relevant evaluative and analytical strategies. Evaluation is a result of both inquiring and reflecting thought processes, i.e. mental activity intrinsically dependent upon a demonstrated, contextually-dependent desire to explore a certain problem space. Analysis is an inquiry into the assumed-to-be unknown and/or a questioning of the assumed-to-be known. Evaluation, is a consolidating process, where judgments are made, and assumed 'truths' and 'knowledge' are incorporated into some kind of hierarchy. Together, an analysis (i.e. creation of 'new' knowledge) and evaluation (i.e. categorization of 'existing' knowledge) represent closing of a learning circle. Any conscious reflection over requirements for a higher quality learning circle could become a daunting exercise as it involves raising the quality of 'knowing'. This is why a framework such as SST has an important role to play.

SST involves three aspects intra-analysis, inter-analysis and value-analysis. These should not be regarded as sequential, as it is possible to begin at any point in the framework. SST is intended to be iterative, and therefore it is possible to move from one analysis to another repeatedly and in any direction, at any time. A range of methods are available to the actors, and their facilitating external analyst, in seeking 
to articulate their worldviews. These methods include: rich pictures, brain-storming, mind-maps, diversity networks, drama transfers, role-playing - all of which are supporting creation, visualization, and communication of mental models and narratives. Each of the three aspects of the framework helps to guide inquiries with a number of themes. The purpose of intra-analysis is to enable creation of an individual process for structuring a problem. This analysis aims to create and capture a range of narratives from participating stakeholders by providing an enrichment and visualization process for them. Inter-analysis is the aspect of the inquiry which represents collective reflections of decision-making alternatives. The aim is to have a dialogue and to reflect upon ranges of narratives derived through intra-analysis. The purpose is not to achieve consensus or to establish common ground, but to produce a richer base upon which further inquiry and decision-making could proceed. Grouping of narratives takes place through consideration and discussion of individually produced narratives. Results of these inquiries might be considered to form a knowledge base relating to problem spaces under investigation. A critical and reflective approach in considering these results is needed to ensure a basis for 'good' decision-making and to avoid unintended, negative consequences for actors and organizations concerned. Evaluation could be said to be an examination of the 'known' - what has been learned from analyses in a socio-cultural context. Here actors may carry out examinations of values influencing and constraining the analyses, and consider prioritization from political and cultural perspective.

SST can be explained as involving groups of professional members of organisations to act as analysts of their own problem spaces under guidance of expert analysts as external facilitators. This includes examination of their activities and specific use of methodologies, rhetoric and strategies to construct local arguments and findings. By the end of an initial analysis, analysts (e.g. organisational actors) might for example be familiar with some of the strategies available within their organization for further inquiries into contextual dependencies. SST is complementary, rather than alternative, to traditional approaches to analysis. However, there may be conflicts relating to unproblematized assumptions of ontological beliefs and logical empiricism (i.e. unquestioned beliefs of 'objectivities and truths'). Other assumptions may also arise which are incompatible with the underlying philosophy of SST, e.g. the traditional communicational theories, focusing on a 'sender-receiver' perspective. To give a simplified example, in a traditional approach, inquiry might ask what a company wants to achieve with its information and communication system. On the other hand, a contextual inquiry would ask what the people who will use the system want to achieve, and what roles and specific purposes their activities might have in organizational contexts. What makes their unique situation recognizable for them? What specific role do they give to information (and the organizational business)? This inquiry is to be seen as investigation by users themselves into their own assumptions and needs within the space of an open information system (an 'organization', human activity system or socio-cultural system). This is a bottom up perspective on organisation, information and (technical) communication systems. Systems are envisaged, which are shaped with the intention to serve specific organizational actors and their needs - from their own points of view.

\section{Summary}

Contextual inquiry is intended to support analysts to recognize individual emergence, multiperspectivity and open systems thinking in combination. Two different 
categories of emergence are highlighted. In the first, each individual's identity is an emergent property of a number of emergent systems of which the individual is a member. In the second category, each organization is an emergent property of the multiple perspectives of all the interacting individuals for whom its existence is relevant. There are multiple views of what comprises the organization, formed from the multiple perspectives of many individuals. From a systems analyst's point of view, many possible descriptions will emerge in any organizational inquiry, through the differing experiences of context among many individuals. The boundaries of an organizational system will be dependent upon multiple perspectives and descriptions from individuals. This requires consideration to be given to sense-making, emotion and learning processes that those individuals engage in. It is helpful to highlight different levels of abstraction involved in discussions about systems as emergent properties of socio-cultural phenomena.

The Strategic Systemic Thinking framework is discussed as a contemporary version of contextual analysis. Its aim is to support application and use of specifically adapted methods by groups of individual stakeholders in their efforts to construct understanding and meaning. Its focus is on ways in which information needs and information use are created by individuals. A concept of contextual dependency is of interest because it supports a focus of inquiry by unique individuals, on their own individual beliefs, thoughts and actions in specific situations and contexts. Through this kind of inquiry support is provided for a contextually-dependent creation of necessary knowledge. This has potential to provide a foundation for more successful communication, systemic analysis and eventually information systems development to be achieved. The purpose is to create a form of organizational transformation that allows individual emergence to surface.

\section{References}

Ackoff R.L. (1999). Ackoff's Best: His Classic Writings on Management. NY: Wiley Argyris C. (1990). Overcoming Organizational Defenses: Facilitating Organizational Learning. Englewood Cliffs, New Jersey: Prentice Hall.

Argyris C. \& Schon D. A. (1978). Organizational Learning. Reading Mass: Addison Wesley. Argyris C. \& Schon D. A. (1996). Organizational Learning II - Theory, Method and Practice. Reading Mass: Addison Wesley.

Bateson G. (1972). Steps to an Ecology of Mind. University of Chicago Press.

Bateson G. (2003). Mind and Nature: a Necessary Unity. Cresskill, J: Hampton Press.

Bednar P. M. (2000). A Contextual Integration of Individual and Organizational Learning Perspectives as Part of IS Analysis. Informing Science, Journal. p145 - 156. Volume 33.

Bednar P.M. (2007). 'Individual Emergence in Contextual Analysis.' Special Issue on Individual Emergence, Systemica (Journal of the Dutch Systems Group), 14 (1-6) 23-38

Bednar P.M. and Welch C. (2007). 'A double helix metaphor for use and usefulness in Informing Systems,' in Special Series of Informing Science Journal 'A double helix relationship of use and design in IS?', H-E. Nissen, P.M. Bednar and C. Welch (series editors), Forthcoming, 2007.

Berger P. L. and Luckmann T. (1966). The Social Construction of Reality: A Treatise in the Sociology of Knowledge. Anchor Books.

Bertalanffy L. von (1969).General Systems Theory. NY: George Brasiller.

Boulding K.E. (1953). The Organizational Revolution. NY: Harper \& Row

Checkland P. (1981). Systems Thinking, Systems Practice. Chichester: John Wiley \& Sons.

Checkland P. \& Holwell S. (1998). Information, Systems and Information Systems - making sense of the field. Chichester: John Wiley \& Sons. 
Churchman C.W. (1968). The Systems Approach. NY: Delacourt Press.

Ciborra C.U. (2004). Getting to the Heart of the Situation: The Phenomenological Roots of Situatedness. Interaction Design Institute, Ivrea, Symposium 2005. Accessed June 2007 at: http://projectsfinal.interaction-ivrea.it/web/2004 2005.html

De Zeeuw G. (2007). Foreword to Special Issue on Individual Emergence, Systemica (Journal of the Dutch Systems Group), 14 (1-6) ix-xi

Emery F.E. (1969). Systems Thinking. Harmondsworth: Penguin Books Ltd

Fayol, H. (1949). General and industrial management. London. Pitman Publishing company

Hay N. (2007). Image as a Therapeutic Catalyst. The Systemist, 29(1) 7-20.

Klein H.K. (2007). Fourth Leverhulme Lecture, January 12 2007, Salford Business School, UK

Langefors B. (1966). Theoretical Analysis of Information Systems. Lund: Studentlitteratur.

Langefors B. (1995). Essays on Infology - Summing up and Planning for the Future. Lund: Studentlitteratur.

Levy D.L., Alvesson M. and Willmott H. (2003). Critical Approaches to Strategic Management., in Studying Management Critically, Alvesson M. and Willmott H., editors, Sage: London

Maturana H. R. and Varela F. J. (1980), Autopoiesis and Cognition: The Realization of the Living. Reidel.

Minati, G. (2006). Call for Papers, AIRS Congress 2007, http://www.airs.it/AIRS/indexEN.htm, accessed 18 September 2007

Nissen H.E. (2007). 'Using Double Helix Relationships to Understand and Change Informing Systems,' Special Series of Informing Science Journal: A double helix relationship of use and design in IS? (forthcoming)

Radnitzky G. (1970). Contemporary Schools of Metascience. Gothenburg: Akademiforlaget.

Shannon C.E. and Weaver W. (1999). The Mathematical Theory of Communication. University of Illinois Press.

Schein E (1992). Organizational Culture and Leadership, 2nd edition, Jossey-Bass.

Senge P.M. (1990). The Fifth Discipline: The Art \& Practice of the Learning Organization. Doubleday: New York

Simon H.A. (1969). The Sciences of the Artificial. Cambridge, Mass.: MIT Press.

Sorokin P. (1928). Contemporary Sociological Theories, NY: Harper

Ulrich W. (1983). Critical Heuristics of Social Planning. Chichester: Wiley.

Ulrich W. (2001). 'Critically Systemic Discourse: A Discursive Approach to Reflective Practice in ISD', Parts 1 \& 2, The Journal of Information Technology Theory and Application (JITTA), 3.3, 2001, pp 55-106.

Vickers G. (1970). Freedom in a Rocking Boat. Allen Lane.

Weick K. (1995). Sensemaking in organizations. Thousand Oaks, Cal: Sage Publications. 